J3eA, Journal sur l'enseignement des sciences et technologies de l'information et des systèmes, Volume 3, Hors-Série 1, 20 (2004)

DOI : http://dx.doi.org/10.1051/bib-j3ea:2004620

(C) EDP Sciences, 2004

Maisons intelligentes pour personnes âgées : technologies de l'information intégrées au service des soins à domicile

N. Noury, G. Virone, P. Barralon, V. Rialle et J. Demongeot

Laboratoire TIMC-IMAG-CNRS 5525

Faculté de Médecine de Grenoble F-38706 La Tronche, France

Norbert.Noury@imag.fr 


\title{
Maisons Intelligentes pour Personnes âgées : Technologies de l'Information Intégrées au Service des Soins à Domicile
}

\author{
Norbert Noury, Gilles Virone, Pierre Barralon, Vincent Rialle, Jacques Demongeot \\ Laboratoire TIMC-IMAG-CNRS 5525, \\ Faculté de Médecine de Grenoble, 38706 La Tronche, \\ Tel 0476637486, Fax 0476518667 \\ Norbert.Noury@imag.fr
}

\begin{abstract}
Résumé - Le concept d'Habitat Intelligent pour la Santé vise à redonner une vie autonome, dans leur domicile, à des personnes qui devraient normalement être placées dans des établissements spécialisés : patients souffrants de certaines maladies chroniques, handicapés, mais aussi personnes âgées dépendantes. Cet article propose un état de l'art du concept des habitats intelligents pour la santé. Il présente ensuite les principales démarches menées dans les domaines technologiques concernés - systèmes d'informations, équipements domotiques, capteurs ubiquitaires, assistance robotisée - les essais de standardisation menés par quelques consortiums, et enfin quelques réflexions éthiques.
\end{abstract}

Mots clés : Télésurveillance Médicale, Domotique, Maison Intelligente, Assistances Techniques, Systèmes d'Informations.

\section{INTRODUCTION}

L'évolution démographique va dans le sens d'une population plus âgée. Les taux de fertilité en Europe, en Asie orientale, au Canada, sont tous autour ou en dessous du seuil de remplacement de 2,1 enfants par femme. Cela entraîne un taux de croissance négatif de la population, donc une structure de population de plus en plus âgée. En l'an 2035 un tiers de la population des pays développés devrait avoir plus de 65 ans (source OMS).

Une telle évolution va alourdir les systèmes de santé des pays concernés autant sur le plan économique que social. Les coûts économiques seront directement liés aux soins eux mêmes, mais intégreront également les coûts « cachés » de réorganisation des systèmes de soins : à cause de l'importante population de personnes nécessitant des soins, il faudra complètement réorganiser le système de distribution de soins et redéfinir les tâches entre les différents acteurs du réseau de santé. La pression sociale s'intensifiera aussi car les citoyens souhaiteront jouer un plus grand rôle dans la prise en charge de leur santé, réclameront une information plus complète sur leur état de santé, et souhaiteront également une plus grande autonomie dans la prise en charge de leur santé. Cette charge de travail accrue dans le secteur de la distribution des soins, provoquera une pénurie en personnels qualifiés. Aussi, pour rendre attractifs les métiers aux services des personnes âgées, il faudra fournir aux professionnels des outils adaptés, et valorisants, pour améliorer leur productivité et la qualité de leur service.

La télémédecine est maintenant largement acceptée comme un moyen privilégié d'accès à la santé pour tous, et qui peut relever les challenges précédemment exposés. C'est particulièrement vrai pour ces « Habitats Intelligents en Santé » qui intéressent les personnes fragilisées par les maladies, par les handicaps ou par l'âge.

Cet article brosse une revue de ce nouveau champ de la télémédecine, donnant un aperçu des nombreux domaines scientifiques et techniques qu'il adresse, des questions d'usages qu'il soulève aussi, d'éthique ou d'économie avec tous les espoirs et toutes les angoisses qu'il transporte.

\section{LES SYSTEMES DE TELEMEDECINE ET LE DOMICILE - DEFINITIONS ET ETAT DE L'ART}

Dans les 2 dernières décennies, la télésurveillance médicale (Home Health Care) a généré beaucoup d'investigations et abouti à quelques développements de systèmes centrés sur le domicile. Cette télésurveillance médicale repose sur les techniques organisationnelles et les technologies des systèmes d'information, pour tenter d'assurer à domicile un niveau de soins au moins équivalent à celui dispensé en institution. Elle ouvre aussi la voie vers une surveillance médicale plus continue et plus précise, donc plus efficace. Mais il lui faut en plus garantir le respect de la vie privée de la personne et ne pas perturber son mode de vie.

Les caractéristiques requises par de tels systèmes ont depuis très longtemps été identifiées [1,2]. Ces systèmes devront être ouverts, capables d'intégrer des technologies assez diverses, et suffisamment flexibles pour s'adapter au cas particuliers de chaque patient, tout en prenant en compte l'aspect dynamique de l'évolution de l'état de santé.

Le concept d'habitats intelligent en santé est issu de celui des habitats intelligents (Smart Homes) qui intègrent déjà des capteurs et des actionneurs pour "suivre » les occupants, communiquer entre eux, et seconder intelligemment les occupants dans l'accomplissement des tâches journalières et répétitives. Dans le cas du suivi médicale de personnes fragiles, il s'agit de surveiller l'état de santé et le niveau d'autonomie par référence à un état répertorié (par exemple la grille «AG-GIR» [3], les «Activity of Daily Living - ADL » [4]), de détecter les déplacements et les chutes, de remédier à la détérioration des facultés cognitives (perte de mémoire, errances), de veiller aussi à la sécurité de la personne en monitorant les paramètres de son environnement. Enfin il s'agit 
d'assister la personne dans l'accomplissement d'un certain nombre de tâches au travers d'aides techniques.

C'est probablement Togawa et al [5] qui fut l'un des premiers à monitorer les activités journalières du sujet en même temps que certains paramètres physiologiques pour les transmettre vers le personnel médical. Inada et al [6] a rajouté le contact avec l'équipe de secours et l'enregistrement d'informations plus subjectives. Noury [7] s'appuie le premier sur les nouvelles technologies de l'information et de la communication (technologie VideoTex) pour mettre en réseau les domiciles et collecter les variables physiologiques en même temps que des informations textuelles concernant le patient. Dans son " Adaptable Smart Home », Richardson [8] met en place un réseau pour contrôler et monitorer des dispositifs à l'intérieur du logement comme depuis l'extérieur. Alyfuku et Hiruta [9] déposent le brevet sur le monitorage passif de la personne. Celler et al [10] mesure le comportement et l'état de santé de la personne à partir de 18 capteurs interconnectés sur un réseau EchelonLonWorks (détecteurs de présence, capteurs de conditions ambiantes, capteurs d'utilisation des appareils ménagers). Chan, Campo et Steenkeste [11] au CHU de Toulouse, divisent la surface d'une chambre hospitalière en plusieurs zones fonctionnelles, à l'aide de capteurs volumétriques, ils peuvent ainsi monitorer précisément l'environnement du patient et, par un apprentissage par réseau de neurone, détecter de manière automatique certains évènements (sorties de lits, fuites du patient, visites de l'infirmière). L'expérience « Sahal » est décrite par Roth et al [12], il s'agit de télésurveillance à domicile de malades cardiaques. Leikas et al [13] décrit un système pour le monitorage des personnes démentes à domicile en utilisant de simples contacts de portes. La «Neural Network House » de Mozer [14] utilise les réseaux de neurones pour apprendre les réglages environnementaux préférés des occupants et les utilise pour optimiser le système de gestion d'énergie tout en satisfaisant les exigences de l'utilisateur. Williams et Doughty [15] ont développé, dans le cadre du projet «Carernet», une architecture générique de système d'information qui a été implémentée dans le projet « Midas » [16]. Elger [17] met l'accent sur l'assistance technique aux personnes à mobilité réduite, ou souffrant de déficiences visuelles, auditives ou cognitives, dans le démonstrateur «SmartBo » installé dans un appartement ordinaire. Le projet "Assistive Interactive Dwelling (AID) House » de Bonner [18] est l'occasion de s'intéresser aux problèmes d'éthique concernant la surveillance continuelle de la personne. Van Berlo [19] prend en compte les 71 recommandations du label "Dutch Senior Citizen Label" pour concevoir la "Smart Model House for Senior Citizen" insistant sur la sécurité et sur le confort et les économies d'énergie. Le système de Sixsmith [20] bâtit un profil moyen d'activité à partir duquel il peut détecter les écarts au modèle et fabriquer automatiquement des messages d'alertes. Glascock et Kutzik [21] détectent automatiquement les ADL, sans déclenchement d'alarme. Noury et Rialle [22] ont construit au sein de la Faculté de Médecine de Grenoble un véritable appartement appelé
« Habitat Intelligent pour la Santé » (HIS), câblé d'un réseau domotique de type CAN, sur lequel sont connectés les capteurs et actionneurs (Figure 1) qui coopèrent entre eux et avec un système d'information reposant sur les technologies WEB et JAVA. L'architecture mise en place permet de mettre en réseau plusieurs «Habitats Intelligents pour la Santé (HIS) », recueillir les variables physiologiques et d'activité de la personne, détecter des scénarios de situations anormales ou de crises à l'aide d'un système distribué à base d'agents intelligents (Figure 2), et d'agir sur l'environnement du domicile. Maglaveras [23] a développé, dans le cadre d'un projet européen, l'architecture générique du système «Citizen Healthcare System (CHS) », en s'appuyant sur les technologies de programmation orientées objets, les réseaux locaux sans fils, et les standards d'échanges de dossiers électroniques médicaux (DICOM, SCP-ECG). Plus récemment les grands laboratoires de recherche et des industriels de dimensions internationales se sont intéressés à ce domaine et proposent leurs propres projets: Aware House du Georgia Tech Institute [24], « MIT's House of the future" [25], «Assistive Cognition »à l'Université de Whashington [26], «Independent LifeStyle Assistant (ILSA) » de la compagnie Honeywell, « Center for future Health » de l'Université de Rochester-NY [27].

Mais parmi tous ces projets, bien peu ont effectivement été évalués chez les personnes âgées et encore moins sont arrivés au stade commercial.

\section{LE SYSTEME D'INFORMATION POUR LA TELESURVEILLANCE MEDICALE}

Le système d'information organise la circulation des informations relatives au patient, il doit permettre la prise en charge par des acteurs distants et distribués. Son analyse passe par une modélisation. L'outil actuellement reconnu en matière de modélisation des systèmes d'information est le langage «Unified Modelling Language (UML) ».

\section{A. Acteurs, données et cas d'utilisation}

Les acteurs du système sont: le patient lui-même, les producteurs de soins (médecins hospitaliers, médecins de ville, infirmières et aides soignants), les pharmaciens, les laboratoires d'analyses biologiques et de radiographies, les téléopérateurs (service $24 \mathrm{~h} / 24 \mathrm{~h}$ ), les travailleurs sociaux (assistante sociale, aide ménagère, service de livraison des repas), les utilisateurs occasionnels (membres de la famille, voisins), les personnels d'urgence, et les administrateurs du système (soutien technique et logistique).

Les différentes fonctionnalités du système concernent (Figure 2): l'identification dans le système, la consultation des dossiers médicaux, la rédaction ou la consultation des prescriptions, l'écriture et la lecture des paramètres. Les acteurs au domicile (soignants, travailleurs sociaux) peuvent être amenés à ajouter des notes d'intervention. Les administrateurs ont leur propres 
cas d'utilisations (mise à jour de logiciels et de matériels, modifications des paramétrages).

Enfin on identifie divers types de données: les prescriptions rédigées par les médecins, les protocoles de surveillance (choix des données, des capteurs et des instants d'enregistrement), les paramètres vitaux (valeurs, instants de prise, qualité de la prise), les commandes (en direction des machines et des actionneurs au domicile), les messages échangés entre les acteurs (messagerie), les identifiants personnels (droits d'accès et d'actions aux données), et enfin le dossier médical qui regroupe toutes les informations administratives concernant le patient, l'identification des services dont il dépend, et tout l'historique des prescriptions, des paramètres vitaux enregistrés, des résultats d'analyses médicales. Certaines informations sont confidentielles.

\section{B. Architecture logique}

Le système d'information comprend des postes patients (système domotique), un serveur, et des postes clients (Figure 4).

\section{B.1. Le serveur}

Le serveur est constitué d'une base de données, d'un serveur de communication et d'un module d'aide à la décision :

- La base de données mémorise toutes les informations pertinentes du patient: le dossier médical, les données saisies au domicile par le patient et les capteurs qui l'entourent, les alarmes générées, les notes des médecins, les remarques médicales, les notes des infirmières, les messages du patient et des intervenants.

- Le serveur gère un accès sécurisé à la base de données au travers du réseau.

- Le module d'aide à la décision analyse « en temps réel » le trafic des données entre les intervenants.

Les composants du serveur peuvent être répartis entre plusieurs ordinateurs. Le serveur peut fonctionner de manière autonome ou bien en collaboration avec le système d'information médical.

\section{B.2. Le Poste patient}

Le poste patient est situé au domicile, il comporte :

- Un module de communication avec le serveur, qui repose sur divers types de liaisons (modem téléphonique, ADSL, ISDN), et divers protocoles disponibles.

- Des modules spécifiques pour l'acquisition des données.

- Un module de calcul pour l'analyse des données, l'évaluation du patient, la prise de décisions en autonome (indépendamment de la connexion distante) et la génération d'alarmes de $1^{\text {er }}$ niveau. Cela suppose l'existence d'une base de données locale.
- Un module de présentation des données : interface patient conviviale pour la présentation, et la saisie éventuelle, des données. Il peut reposer sur une large variété de matériels : écrans LCD, PC, Palm Top, TV.

Le poste patient peut être une unité autonome ou bien une combinaison de dispositifs interconnectés.

\section{B.3. Le poste client}

Le poste client, utilisé par les professionnels de santé pour accéder aux données patients, ajuster la thérapie, visualiser les alarmes, échanger des messages avec les autres participants, comprend :

- Le module de communication qui permet de communiquer avec le serveur WEB (navigateur).

- Le module de présentation qui dépend du type de client, de sa profession, de sa fonction dans le système, de sa spécialité.

Le poste client peut être implémenté sous diverses formes: ordinateur personnel, téléphone cellulaire portable, etc.

\section{B.4. Module d'aide à la décision}

Le module d'aide à la décision analyse l'impact de la thérapie sur le patient. Grâce à un échantillonnage plus fréquent que le suivi habituel (visites mensuelles chez le médecin, courts séjours en hospitalisation) il permet une analyse plus fine, même si les données sont éventuellement moins fiables en valeurs absolues (dans ce cas c'est la « tendance » des données qui est intéressante). Il permet également une analyse de l'évolution globale et conjointe des divers paramètres patients (fusion de données). Ce module devrait être distribué entre le serveur et le poste patient afin de bénéficier d'un support même en l'absence de connexion.

Le poste patient effectue une validation des données et offre une interface pour l'information du patient. Le serveur effectue des traitements plus complexes sur les données : chaque fois qu'il reçoit des nouvelles données, il les analyse à la lumière des données antécédentes (adaptation au patient) et en fonction de certaines règles pour déterminer des scénarios prédéterminés. Ce module pourrait lui même devenir un agent intelligent d'un système d'analyse épidémiologique en temps réel.

\section{EQUIPEMENTS DOMOTIQUES POUR LE DOMICILE}

L'équipement du domicile peut être modélisé par analogie avec le modèle du corps humain qui met en oeuvre une architecture d'intelligence distribuée. On y trouve des fonctions sensorielles (« effecteurs »: capteurs), d'actions et de réactions («effecteurs»: actionneurs), et des fonctions intégratives qui réalisent l'analyse de l'information, le stockage et les prises de décisions. Le système nerveux central (encéphale et 
moelle épinière), s'appuyant sur les axones, se charge des activités conscientes et des décisions vitales, ainsi que du traitement de toutes les informations qui requièrent une importante charge de traitement (vision, ouie, odorat). Parallèlement, le système nerveux périphérique (nerfs crâniens et périphériques) se charge de la communication avec les neurones afférents et efférents, et gère les activités réflexes (respiration, réaction à la sensation de brûlure). Le second système développe deux moyens de communication plus élaborés, basés sur les réseaux sympathiques et parasympathiques, des communications physiques au travers des ganglions et en même temps des liaisons non-physiques à l'aide des neuromédiateurs chimiques.

De même dans l'équipement du domicile on va retrouver des capteurs et des actionneurs, des réseaux de communication, et des dispositifs intelligents distribués pour s'occuper des fonctions réflexes (fonctions domotiques, alarmes) et des fonctions centrales (détection des modes d'activité, déclenchement des alertes).

\section{A. Les Capteurs}

Les données permettant d'évaluer l'état de santé de la personne sont évidemment ses données physiologiques, mais elles sont utilement complétées par la détection de ses activités et postures, en même temps que le monitorage de son environnement. Les importants progrès scientifiques et techniques effectués en instrumentation miniaturisée, en capteurs et en traitement du signal, ont permis d'élargir le champ d'action de l'instrumentation biomédicale aux domaines sportifs et grand public, et pour des tâches non directement médicales : les mesures non invasives ont ainsi permis de rapprocher l'acquisition du patient de celle de son environnement [28].

\section{A.1. Capteurs des données physiologiques}

Les signes vitaux essentiels sont bien identifiés depuis longtemps par les médecins et la liste des signaux accessibles depuis le domicile n'est pas limitée.

Le poids se prête aisément à une " auto mesure », comme les pèse personnes du commerce sont d'une utilisation bien connue. On peut envisager l'automatisation de la prise de cette information, par exemple en pesant la personne dans son lit [29] ou dans les toilettes [30].

La température centrale s'acquiert facilement avec les thermomètres électroniques qui mesurent le rayonnement infrarouge de la surface du tympan (thermomètres tympaniques) ou du fond de la bouche. Ils ont totalement remplacé les dispositifs au mercure qui sont désormais interdits à cause de la toxicité du métal qu'ils renferment.

Les valeurs extrêmes de la tension artérielle (pressions systolique et diastolique), sont accessibles avec des dispositifs à brassards (sphygmomanomètres) qui se gonflent automatiquement. Ils nécessitent de respecter certaines conditions lors de la mesure (position de repos, compensation de la différence de hauteur entre le cœur et le site de mesure).

La saturation en oxygène du sang (Oxymétrie) permet d'évaluer de manière non invasive la qualité de la ventilation. Le procédé optique mesure l'opacité du sang aux extrémités des doigts, des orteils ou du lobe de l'oreille. Si cette valeur, en instantané, ne présente pas réellement de sens médical, son monitorage en continu, en particuliers pendant le sommeil, peut permettre de détecter et de quantifier des anomalies telles que les variations excessives de fréquence, ou les apnées obstructives du sommeil.

Le pouls s'obtient de plusieurs manières. A partir du signal électrique cardiaque («Electrocardiogramme ECG »), on peut déterminer l'intervalle de temps séparant 2 signaux typiques (complexes «QRS»). La période entre 2 battements cardiaques est plus simplement mesurable sur le signal acoustique amplifié par un stéthoscope. Enfin, comme la fonction du cœur est de pousser périodiquement le sang chargé d'oxygène dans le système vasculaire périphérique, on peut remonter à la fréquence cardiaque soit en évaluant la distance entre les instants successifs des pressions artérielles systoliques et diastoliques (tensiomètre), soit entre les extremums du taux d'oxygénation du sang (oxymètre). Toutefois le pouls instantané est rarement intéressant, sauf si il dépasse ses limites inférieures (quelques battements par minute) ou supérieures (quelques battements par seconde), par contre son enregistrement en continu permet d'observer les fluctuations.

Bien d'autres capteurs, également disponibles dans le commerce, permettent d'accéder à d'autres paramètres plus spécifiques de certaines pathologies : taux de glycémie chez les diabétiques (Glucomètre), capacité respiratoire chez les asthmatiques (PeakFlow meter).

\section{A.2. Capteurs d'activité}

Il s'agit soit de détecter les stationnements et les déplacements $\mathrm{du}$ sujet dans son environnement (référentiel externe), soit de détecter ses postures (référentiel interne) et des évènements tels que la chute.

Le sujet peut être repéré par des détecteurs volumétriques qui mesurent le rayonnement Infrarouge émis par la surface du corps ou bien qui détectent les ondes ultrasonores réfléchies. De simples détecteurs de passages aux portes (contacts) permettent aussi de localiser le sujet, tandis que l'installation de capteurs au sol permet de situer le sujet avec plus de précision et de distinguer la présence d'un animal de compagnie.

Les postures corporelles (debout, penché ou allongé) peuvent être déterminées simplement avec un inclinomètre à bille (l'usage du mercure est maintenant prohibé), mais l'information délivrée par un accéléromètre à un ou plusieurs axes permet une plus grande précision sur l'inclinaison du corps, sa vitesse et son accélération [31] et permet même de détecter la chute du porteur [32]. Les sols actimétriques sont également 
très prometteurs en ce sens puisqu'ils permettraient de distinguer une personne debout ou allongée par une simple différence de surface occupée au sol.

\section{A.3. Conditions environnementales}

Il est enfin nécessaire de monitorer les grandeurs environnementales du domicile car ce sont les grandeurs d'influence sur les autres mesures, mais également parce qu'elles nous renseignent sur le mode de vie du sujet : la température dans les pièces (thermomètre), la pression atmosphérique (baromètre), l'humidité relative (Hygromètre), la luminosité (Luxmètre), le niveau de bruits (sonomètre) [33].

Certains paramètres d'environnement concernent également la sécurité du sujet: CO, gaz, (détecteurs de gaz), feu (détecteur d'incendie).

\section{B. Actionneurs et machines}

Le système d'appartements intelligents peut aisément profiter des technologies existantes en domotique pour faciliter l'accomplissement de certaines tâches par la personne. Par exemple, déverrouiller une porte en cas d'urgence, allumer ou éteindre les lumières, régler le niveau de chauffage.

De plus des robots peuvent venir en aide à la personne pour effectuer certaines tâches journalières («Assistive robotics»). Ainsi un bras manipulateur peut être monté soit sur la chaise roulante, soit sur un robot mobile, pour appuyer sur un bouton de commande, manipuler un levier de porte ou bien s'emparer d'un objet. Des tâches plus élaborées peuvent utilement être réalisées par ces robots aidants : préparer un repas ou faire le ménage [34], faire sa toilette [35]. Ces robots peuvent être pilotés par la voix ou le geste [36].

Egalement, en fonction des pathologies, le système HIS devra prendre en compte des «machines thérapeutiques » (pousse seringue, perfusion, machine de dialyse, piluliers) qui peuvent également participer à la tâche d'acquisition de données par leurs propres capteurs.

\section{Réseaux locaux}

Les réseaux locaux font appel à des réseaux câblés domotiques (bus de terrains) ou bureautiques (Ethernet, USB), cependant les réseaux sans fils (ISM $868 \mathrm{MHz}$, IEEE 802.11 et Bluetooth) semblent évidemment promis à un plus grand avenir puisque, pour des bandes passantes équivalentes, ils ne nécessitent pas un câblage définitif et sont sans impact sur l'architecture du bâtiment.

Le critère de choix du réseau local est rarement uniquement celui de la bande passante disponible, par contre on attend un service en temps réel, une prise en charge des collisions, une détection et une correction des erreurs pour ne pas rater l'occurrence d'une donnée vitale mais fugace. Par exemple le projet HIS de Grenoble utilise le réseau CAN [37] qui présente une bande passante réduite $(1 \mathrm{Mbps})$ mais permet un service de type " producteur-consommateur » qui convient très bien à la diffusion d'informations et aux boucles intelligentes distribuées. En outre, il s'adapte à tous types de médium de communication: dans ce projet il emprunte indifféremment une simple paire téléphonique et une passerelle sans fils (bande ISM à $868 \mathrm{MHz}$ ).

\section{Agents intelligents}

Les agents (artificiels) intelligents sont des entités actives qui possèdent des facultés de perception, de raisonnement et d'action sur leur environnement. Dans le cas présent, ces agents sont chargés du monitorage et de l'assistance à distance de la personne. Ils remplissent des rôles à divers niveaux du système: contrôle de la cohérence des informations, détection des modifications de l'état de santé de la personne, détection d'évènements anormaux contextuels (alarmes), aide au diagnostic, aide à la prise en charge, aide à la planification thérapeutique. Ils sont également chargés des autotests du dispositif (« Built in tests »).

\section{D.1. Cohérence des données et tests intégrés}

La validité des données doit être effectuée dès leur acquisition pour garantir la cohérence des valeurs et leur cohérence temporelle. Par exemple on peut vérifier l'appartenance d'une donnée numérique à un intervalle d'existence. Pour une donnée textuelle on peut vérifier qu'elle appartient à une collection prédéterminée.

\section{D.2. Alarmes}

La qualité principale d'une alarme est de réduire le délai entre l'apparition d'un symptôme et le déclenchement de l'intervention. La seconde est d'alerter le bon interlocuteur (voisin ou un membre de la famille, le médecin, les pompiers ou le SAMU.

\section{D.3. Détections de signes avancés}

Un des rôles des agents de détection est d'aider le médecin dans sa tâche de détection des symptômes avant coureurs d'une pathologie. De nombreux paramètres sont à disposition, mais la représentation de données brutes n'est ni conviviale ni efficace, il s'agit donc de les fusionner et de les présenter d'une manière telle que la situation soit plus aisément identifiable par le médecin.

\section{E. Interfaces homme-machine}

Les interfaces homme machines doivent être adaptées aux utilisateurs non expérimentés : sans être technophobes, les personnes âgées ont plus de difficultés à appréhender les aides techniques, et les interfaces mal pensées sont rapidement abandonnées. Il faut évidemment adresser en tout premier lieu les problèmes liés aux 
diverses déficiences physiques (vision [38], ouie, difficultés de communication langagière [39]). Les interfaces multimodales sont évidemment les mieux adaptées [40]. On peut recourir à tous les types d'interfaces (ordinateur individuel, Palmtop, Téléphone cellulaire) mais les plus acceptables sont encore les plus habituelles (Téléviseur et télécommande).

Ces aspects concernent l' « informatique ubiquitaire »: les ordinateurs intègrent désormais des capteurs qui les renseignent sur l'environnement de la personne et ses aptitudes, et peuvent ainsi adapter dynamiquement le service qu'ils peuvent rendre.

\section{F. Efforts de standardisation}

Comme on l'a dit précédemment, il existe de nombreux projets mais peu d'entre eux ont atteint le stade industriel et ont été commercialisés, par manque de normalisation et de standardisation des solutions technologiques et organisationnelles (interopérabilité).

Toutefois des efforts ont été réalisés dans les domaines de la domotique et des systèmes d'informations, en particulier depuis 1999 avec la création de plusieurs consortiums emmenés par certains grands industriels.

Le consortium UPnP [41] vise à développer une connectivité simple entre des appareils divers et des ordinateurs individuels de diverses origines (interopérabilité). Le consortium OSGI [42] vise à définir et faire adopter des spécifications ouvertes de services pour des réseaux domotiques et des réseaux embarqués dans les véhicules. On trouve ensuite des initiatives dans le domaine des systèmes d'informations avec le consortium «Salutation » [43] (IBM), qui développe une technologie qui permet aux dispositifs de s'identifier préalablement à toute communication, au travers de l'échange de «cartes de visites» qui leur permettent ensuite de se proposer des services adaptés. La seconde grande initiative, la technologie «Jini Network Technology » [44] de SUN, basée sur la technologie JAVA vise à offrir une plateforme de développement commune pour toutes les applications « interconnectées ».

\section{FACTEURS HUMAINS ET ETHIQUES}

Mettre en réseau télé-surveillé une personne, même pour des raisons médicales n'est pas anodin. Un certain nombre de questions sont soulevées par (1) l'observation de la personne à travers «l'œil» des capteurs, (2) la transmission des données d'observation à l'extérieur du domicile privé, (3) l'utilisation de ces données par des tiers. De même faut il veiller à maintenir l'équilibre entre les aspects aidants et des aspects contraignants et éviter les dérives potentielles auxquelles un appareillage de type "écoute" risque de conduire.

Mais il s'agit de savoir si les technologies de télésurveillance médicale au domicile permettront d'installer et de maintenir des conditions de sécurité et d'efficacité médicales comparables à celles dont aurait bénéficié la personne dans des institutions de santé (service hospitalier, clinique, maison de retraite médicalisée, etc.), quel sera le degré d'acceptation, voire d'adaptation, de la personne à son équipement et à sa télésurveillance médicale, et enfin comment s'établira l'équilibre entre deux exigences parfois opposées : celle de la sécurité d'un côté, celle de la liberté de l'autre côté. Il faut également se garder de développer une « médecine des riches » qui développerait une société sous le niveau de protection sanitaire minimum et amplifierait encore la « fracture numérique ».

Mais il faudra bien aussi repenser la place de la personne âgée dans notre société. Avec l'éclatement du noyau familial, les aînés sont de plus en plus isolés en institution ou même à domicile. Devenue plus fragiles, ils peuvent faire l'objet de maltraitances ou même avoir des tendances suicidaires. Une étude canadienne menée sur 2000 aînés relève un taux de $4 \%$ de sujets ayant été victimes de ces violences [45], ce qui est confirmé par le rapport de l'OMS [46] qui avance le chiffre de $6 \%$ des personnes âgées concernées par ces mauvais traitements. Près de 3000 personnes de plus de 65 ans se sont suicidées en 1998 en France [47]. Une réflexion éthique sérieuse doit donc accompagner, voire encadrer, les déploiements de la télémédecine pour les soins à domicile.

Ces questions sont déjà abordées au cours de débats entre les divers intervenants (médecins, malades, associations de malades, aides sociaux, corps infirmier, sociétés de service technique...) dans le cadre déjà actif de l'éthique médicale [48].

\section{CONCLUSIONS}

La télémédecine apporte la promesse d'un meilleur accès aux soins en même temps qu'une réduction des coûts.

Elle transporte aussi des aspects potentiellement dangereux, cependant il est important de mettre en relief les aspects éminemment positifs des technologies de maintien au domicile de personnes fragiles [49]. Les personnes atteintes dans leur corps et leur vie familiale et sociale (handicapés, personnes à risque de chute, insuffisants rénaux dialysés, insuffisants cardiaques et respiratoires, etc.) connaissent un désarroi que nos moyens techniques peuvent contribuer à alléger.

Sur le plan technologique ce domaine souffre actuellement d'une idée forte selon laquelle la médecine nécessite obligatoirement des hautes technologies. En particuliers, les grands opérateurs et constructeurs de télécommunications tentent d'imposer leurs solutions technologiques sans bien comprendre les réels besoins. Or on peut affirmer que des systèmes d'information basés sur les briques de base d'Internet suffisent (SMTP, HTTP).

$\mathrm{Du}$ point de vue scientifique et technique, de grandes avancées restent à accomplir vers de nouveaux signaux qui permettraient de mieux cerner l'état de santé mais également son «bien être ». La contribution d'un grand nombre de «capteurs élémentaires », disséminés 
dans l'environnement de la personne, permettra d'aller vers une acquisition « ubiquitaire » des données («ubiquitous sensing ») en faisant appel à l'approche fusion multi-capteurs adoptée dans les capteurs intelligents (Smart Sensors ») [50].

En ce qui concerne les systèmes domotiques, les recherches doivent s'orienter vers les logements existants où vivent la majorité des personnes.

Sur le plan industriel, L'Habitat Intelligent pour la Santé constitue indéniablement un potentiel important, mais il se développe lentement. Le décollage de ce marché passe probablement par une meilleure prise en compte des besoins des utilisateurs de la part des concepteurs, une grande capacité d'innovation de tous les acteurs -sans forcément faire appel aux technologies de pointe-, des efforts importants pour développer le marché correspondant à ces innovations.

En conclusion, il ne manque pas de technologies pour la télémédecine, mais il manque un modèle d'organisation pour déployer utilement ces technologies.

\section{REMERCIEMENTS}

Nous tenons à remercier le Ministère de l'Education Nationale et de la Recherche Technologique, qui a subventionné le projet «TIISSAD» dans le cadre de l'Action Concertée Incitative 1999 (aide n99B0616). Nous voulons également remercier les industriels qui nous ont offert leur aide technique ou matérielle dans ce projet: ATRAL S.A., ATMEL France, Moulinex, ABSYS S.A. Un remerciement tout particuliers à Monsieur Jean-Pierre THOMESSE, Professeur à l'ENSEM de Nancy, pour ses précieux conseils en matière de réseaux locaux, et à Monsieur André DITTMAR, ingénieur de recherches au LPM-INSA de Lyon pour ses conseils éclairés en matière de capteurs.

\section{BIBLIOGRAPHIE}

[1] SJ Brownsell, G Williams, DA Bradley, R Bragg, P Catlin, J Carlier, "Future systems for remote health care", Jour. Telemedicine \& telecare, vol 5, pp 141-152.

[2] N. Noury, "Système télématique pour l'organisation de l'hospitalisation à domicile", Thèse de doctorat, Université Joseph Fourier de Grenoble, novembre 1992.

[3] JM Vetel, «Le logiciel AGGIR» - Revue hospitalière de France, 1, Jan 1995, p 108

[4] Katz, S., et al., "Studies of Illness in the Aged. The Index of ADL. A Standardized Measure of Biological and Physiological Function." Journal of American Medical Association, 185:914, 1963.

[5] A. Yamaguchi, M. Ogawa, T. Tamura, T. Togawa, "Monitoring behaviour in the home using positioning sensors", in proc. $20^{\text {th }}$ IEEE conference on engineering in Medicine and Biology, pp 1977-79, 1998.

[6] H. Inada, H. Horio, Y. Sekita, K. Isikawa; K. Yoshida "A study on a home care support information system", in proc. Of the 7 th world congress on Medical Informatics, pp 349-353, 1992

[7] N. Noury, P. Pilichowski, A telematic system tool for home health care, in Proc. 14th Annual Intern. Conference of the IEEE-EMBS, Paris-France, Oct 92, part 3/7, 1175-1177

[8] S.J. Richardson, D.F. Poulson, C. Nicolle, "Supporting independent living through adaptable smart home (ASH) technologies", in Human welfare and technologies: papers from the human service information technology applications (HUSITA) conference on information technology and the quality of life and services, pp 87-95, 1993.

[9] K. Alyfuku, Y. Hiruta, "Networked health care and monitoring system", Apr 1995, US Patent 5,410,471 Feb 11, 1993.

[10] B.G. Celler, W. Earnshaw, E.D. Ilsar, L. BetbederMatibet, M.F. Harris, R. Clark, T. Hesketh, N.H. Lovell, "Remote monitoring of the health status of the elderly at home, a multidisciplinary project on aging at the University of New South Wales", International journal of Biomedical Computing, 40:147-155, 1995.

[11] F. Steenkeste, H. Bocquet, M. Chan, E. Campo, "La mise en place d'une technologie pour observer le comportement nocturne des personnes âgées en institution ", Innovation and Technology in Biology and Medicine - revue of Biomedical Engineering (ITBMRBM), 22 :25-30, 2001.

[12] A. Roth, Z. Carthy, and M. Benedek, "Telemedicine in emergency home care--the 'Shahal' experience," $J$ Telemed Telecare, vol. 3, pp. 58-60, 1997.

[13] J. Leikas, J. Salo, R. Poramo, Security alatm system support independant living of demented persons“, proc. Gerontechnology $2^{\text {nd }}$ international Conference, pp 402405, 1998.

[14] M.C. Mozer, "The neural network house: an environment that adapts to its inhabitants", in AAAI Spring symposium on intelligent Environments, pp 110114, 1998.

[15] G. Williams, K. Doughty, and D. A. Bradley, "A system approach to acheiving CarerNet - an integrated and intelligent telecare system," IEEE Transaction on Information Technology in Biomedicine, vol. 2, pp. 1-9, 1998.

[16] K Doughty, Isak R, King PJ, Smith P, Williams G. MIDAS - Miniature Intelligent Domiciliary Alarm System - a practical application of telecare. Proc. 1st Joint BMES/EMBS Conf. Serving Humanity, Advancing Technology. Atlanta, 13-16 October 1999.

[17] G. Elger and B. Furugren, "'SmartBo" - An ICT and computer-based demonstration home for disabled people," presented at Proc. 3rd TIDE Congress : Technology for Inclusive Design and Equality Improving the Quality of Life for the European Citizen, 23-25 June, Helsinki, Finland, 1998.

[18] S. G. Bonner, "Assisted Interactive Dwelling HOUSE," presented at Proc. 3rd TIDE Congress: Technology for Inclusive Design and Equality Improving the Quality of Life for the European Citizen, 23-25 June, Helsinki, Finland, 1998. 
[19] A. Van Berlo, "A "smart" model house as research and demonstration tool for telematics development," presented at Proc. 3rd TIDE Congres : Technology for Inclusive Design and Equality Improving the Quality of Life for the European Citizen, 23-25 June, Helsinki, Finland, 1998.

[20] A.J. Sixsmith, "An evaluation of an intelligent home monitoring system", Journal of telemedicine and telecare, 6:63-72, 2000.

[21] A.P. Glascock, D.M. Kutzik, "Behavioral telemedicine: a new approach to the continuous non intrusive monitoring of activities of daily living" Telemedicine journal, 6(1):33-44, 2000.

[22] N. Noury, T. Hervé, V. Rialle, G. Virone, "Monitoring Behavior In Home Using a Smart Fall Sensor And Position Sensors," in Proc. IEEE-EMBS "Microtechnologies in Medicine \& Biology" Lyon-France Oct 2000, pp. 607-610.

[23] N. Maglaveras, V. Koutkias, S. Meletiadis, I. Chouvarda, and E. A. Balas, "The Role of Wireless Technology in Home Care Delivery," presented at MEDINFO 2001, London, 2001.

[24] C.D. Kidd, R. Orr, D. Abowd, C.G. Atkesson, I.A. Essa, B. MacIntyre, E. Mynatt, T.E. Starner, W. Newstetter, "The aware Home: A living Laboratory for ubiquitous computing research", in proc. $2^{\text {nd }}$ International Workshop on cooperative Buildings - CoBuild'99, Oct 1999.

[25] http://architecture.mit.edu/house n/web

[26] H. Kautz, L. Arnstein, G. Boriello, O. Etzioni, D. Fox, «An overview of the assisted cognition project", in proc. AIII Workshop “Automation as caregiver”, 2002.

[27] http://www.futurehealth.rochester.edu/

[28] A. Dittmar, G. Delhomme, " Microtechnologies et Concepts BioInspirés : Les Potentiels en Biomécanique » Archives of Physiology and Biochemistry, Vol. 109 September 2001, pp. 24-34

[29] E Neuman, "Weighing device for bedridden patients", European patent 0854357 A1, Jul. 22, 1998

[30] E. De Canecaude, "Device for weighing individuals on WC seat", Internat. Patent W085 04472, ОСт.10, 1985

[31] Y Depeursinge, "Device for monitoring the activity of a person and/or detecting a fall...", US patent $n^{\circ} 6201476$ B1, Mar 13, 2001.

[32] N. Noury, «Détecteur de chute d'une personne », Brevet Français 01/12046, Sept. 18, 2001.

[33] D. Istrate, M. Vacher, L. Besacier, E. Castelli, J.F. Serignat "Smart Audio Sensor for Telemedicine", in SOC'2003, Grenoble, Juin 2003.

[34] J.L. Dallaway, R.D. Jackson, P.H. Timmers "Rehabilitation robotics in Europe", IEEE Transactions on rehabilitation engineering, 3(1):35-45, March 1995

[35] M. Topping, "The development of Handy 1, a robotic system to assist severely disabled", in Proc ICORR'99: the International Conference on Rehabilitation Robotics, pp 244-249, 1999

[36] Z. Kazi, S. Chen, M. Beitler, D. Chester, R. Foulds, «Speech and gesture mediated intelligent teleoperation", in Mittal et al., pp 194-210.
[37] G. Virone, N. Noury, JP Thomesse, V. Rialle, J. Demongeot, "A home health information system based on the CAN Field Bus", in FET2003, Aveiro-Portugal, 7-8 Juillet 2003.

[38] A. Arditi, "Effective color contrast: designing for people with partial sight and color deficiencies", technical report, Lighthouse international, 1999, www.lighthouse.org

[39] K.F. McCoy, "Interface and language issues in intelligent systems for people with disabilities », in Mittal et al, pp 1-11.

[40] M. Vallès, F. Manso, M.T. Arredondo, F. Del Pozzo, "Multimodal environmental control system for elderly and disabled people", in proc. IEEE-EMBS, pp516-517, 1996

[41] http://www.upnp.org/

[42] http://www.osgi.org/

[43] http://www.salutation.org/

[44] http://www.jini.org/

[45] $\mathrm{N}$ Migus, «Les mauvais traitements à l'égard des personnes âgées », Centre national canadien d'information sur la violence dans la famille, isbn 0-66298535-4.

[46] Rapport de l'OMS « World report on violence and health » : http://www.who.int/violence injury prevention [47] P. Krémer, «La France en tête des pays européens pour le suicide des personnes âgées ", Le Monde, Feb 5, 2002

[48] C. Hervé «Ethique, Politique et Société: vers une politique de santé publique $»$. Paris, Presses Universitaires de France.

[49] P. Larcher, P. Poloméni «La santé en réseaux». Paris: Masson, 2001.

[50] N. Noury « Du signal à l'Information : le capteur intelligent. Exemples industriels et en médecine». Mémoire d'Habilitation à Diriger des Recherches, Avril 2002,

(http://bibliotheque.imag.fr/bibliotheque/collectionselectroniques/publications/theses/2002/index.html ) 




Fig.1. Le dispositif "Habitat Intelligent pour la Santé », à la faculté de médecine de Grenoble, effectue le monitorage des déplacements de la personne grâce aux capteurs volumétriques disséminés dans chacune des pièces. Il enregistre également les variables vitales de poids, pressions artérielles, pouls, taux d'oxygénation, et des variables de l'environnement telles que la température, l’hygrométrie, la luminosité ou le niveau sonore.



Fig.2. Le Système d'information de l' HIS grenoblois analyse en temps réel les données issues des capteurs et cherche à établir un profil type du sujet afin de détecter des écarts au modèle. 


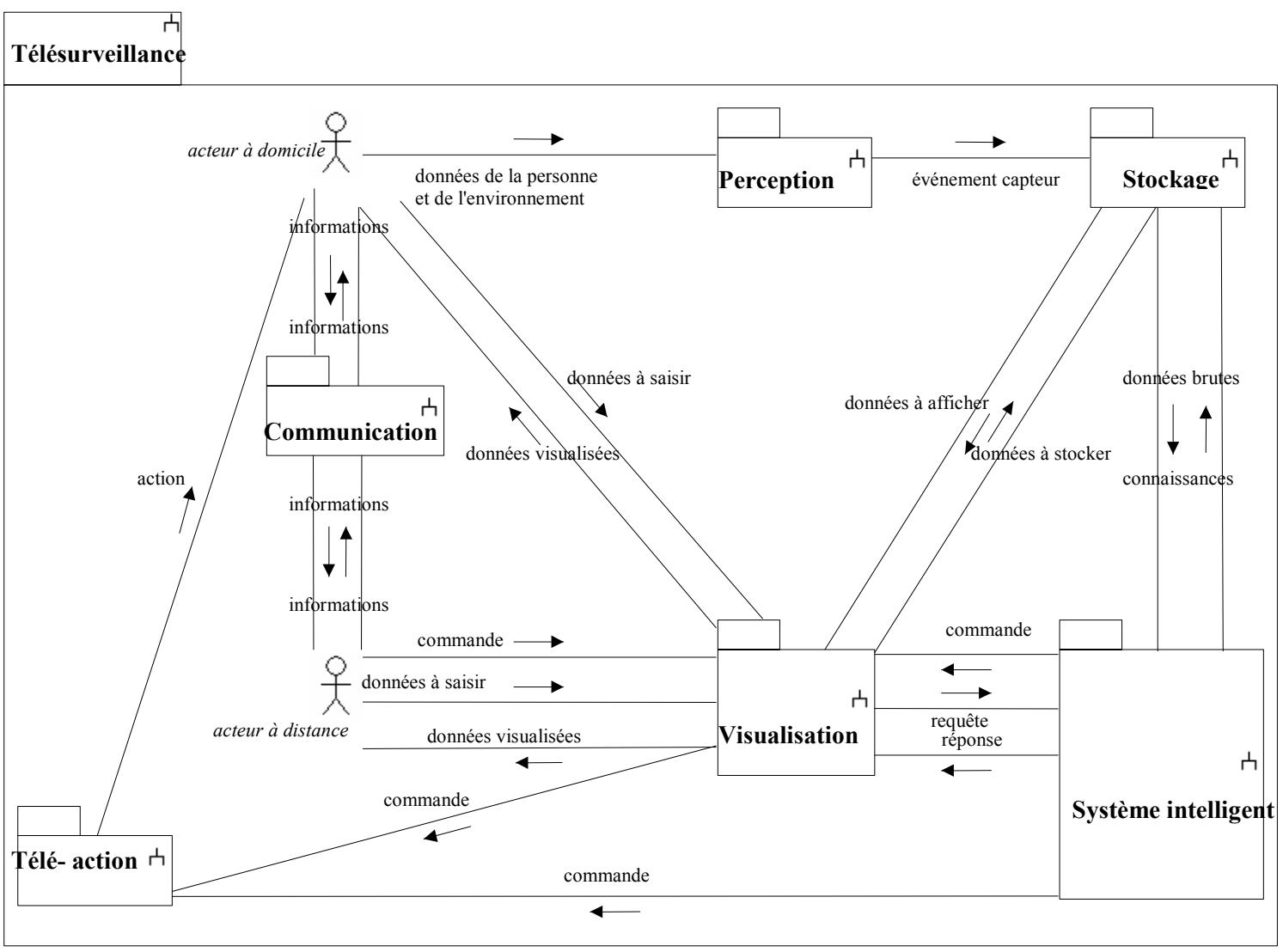

Fig.3. Modèle dynamique du système de télésurveillance - L'acteur à domicile, qui peut être le patient ou bien un intervenant, communique des informations qui sont déposées dans une base de données. L'acteur distant, qui peut être un médecin hospitalier, le médecin traitant ou encore un proche du patient, effectue des requêtes au système intelligent qui se charge de retrouver dans la base de données les données pertinentes et de les mettre en forme pour les visualiser à l'utilisateur. Une interaction directe entre l'acteur domicile et l'acteur à distance peut s'effectuer par le moyen d'une visiophonie. 




Fig.4. Architecture Logique du système d'information - Le serveur d'information est constitué d'un serveur de communication sur Internet, d'une base de données et d'un module de décision. Les postes patients situés aux domiciles permettent l'acquisition des données, les communications avec le serveur et la prise de décisions de premier niveau. Les postes clients communiquent avec le serveur WEB pour émettre des requêtes vers le système gestionnaire de base de données répartie (SGBDR). 